\title{
ANOTHER PROOF OF THE EXISTENCE OF THE ERGODIC HILBERT TRANSFORM
}

\author{
KARL PETERSEN ${ }^{1}$
}

\begin{abstract}
We give a direct proof of the existence of the eigodic Hilbert transform $\sum_{k=-\infty}^{\infty \prime} f\left(T^{k} x\right) / k$, where $T: X \rightarrow X$ is a measure-preserving transformation and $f$ is an integrable function.
\end{abstract}

Cotlar [5], as part of a more comprehensive investigation, has obtained the following result.

THEOREM. Let $(X, \Re, \mu)$ be a measure space, $T: X \rightarrow X$ an invertible measurepreserving transformation and $f \in L^{1}(X, \Re, \mu)$. Then

$$
\tilde{f}(x)=\lim _{n \rightarrow \infty} \sum_{k=-n}^{n} \frac{f\left(T^{k} x\right)}{k}
$$

exists a.e., where the prime denotes that the term with zero denominator is omitted. For $a$ one-parameter measure-preserving flow $\left\{T_{t}:-\infty<t<\infty\right\}$ on $X$, the analogous statement holds:

$$
\lim _{\varepsilon \rightarrow 0^{+}} \int_{\varepsilon \leqslant|t| \leqslant 1 / \varepsilon} \frac{f\left(T_{t} x\right)}{t} d t
$$

exists a.e., for each $f \in L^{1}(X, \Re, \mu)$.

Calderón [4] showed how to derive these results from the existence of the ordinary Hilbert transform. The purpose of this note is to present the most direct proof we have been able to find so far of the a.e. existence of (1). The argument draws heavily on the ideas of Wiener, Loomis, and Calderón.

Other discussions and applications of the ergodic Hilbert transform can be found in $[1,2,6$ and 8$]$.

It is easy to see that (1) exists a.e. in case $f T=f$ a.e. or in case $f$ is a coboundary, that is, a function of the form $f=g-g T$ for some $g \in L^{\infty}$. Since such functions $f$ span a dense set in $L^{1}$, it is enough to show that the set of functions for which the result holds is closed in $L^{1}$. As usual this follows immediately from the corresponding maximal inequality, which is the content of Lemma 1.

Received by the editors May 18, 1982. This paper was presented at the AMS meeting in Toronto on August 26, 1982.

1980 Mathematics Subject Classification. Primary 28D05; Secondary 42A50.

'The author is happy to acknowledge the support of NSF Grant MCS 8001590 and the hospitality of the Laboratoire de Calcul des Probabilités of the Université Pierre et Marie Curie (Universite de Paris, VI), where this work was completed. 
LEMmA 1. There is a constant $c$ such that if $T: X \rightarrow X$ is a measure-preserving transformation on a measure space $(X, \Re, \mu), f \in L^{\mathrm{l}}\left(X, \mathscr{G}_{3}, \mu\right)$, and $\lambda>0$, then

$$
\mu\left\{x: \sup _{n \geqslant 1}\left|\sum_{i=-n}^{n} \frac{f\left(T^{i} x\right)}{i}\right|>\lambda\right\} \leqslant \frac{c}{\lambda}\|f\|_{1} .
$$

Lemma 1 will follow from the analogous statement for the discrete Hilbert transform on $l^{1}$, namely Lemma 2 . This result is well known (see e.g. [7]), but we have not found a direct proof of it in the literature.

LEMMA 2. There is a constant $c$ such that if $\left\{a_{k}\right\} \in l^{1}$ and $\lambda>0$, then

$$
\operatorname{card}\left\{k \in \mathbf{Z}: \sup _{n \geqslant 1}\left|\sum_{i=-n}^{n} \frac{a_{k+i}}{i}\right|>\lambda\right\} \leqslant \frac{c}{\lambda} \sum_{i=-\infty}^{\infty}\left|a_{i}\right| .
$$

Backtracking further, to deduce Lemma 2 it is best first to prove its limit (rather than supremum) version, Lemma 3.

LEMMA 3. There is a constant $c$ such that if $\left\{a_{k}\right\} \in l^{1}$ and $\lambda>0$, then

$$
\operatorname{card}\left\{k \in \mathbf{Z}:\left|\sum_{i=-\infty}^{\infty} \frac{a_{k+i}}{i}\right|>\lambda\right\} \leqslant \frac{c}{\lambda} \sum_{i=-\infty}^{\infty}\left|a_{i}\right| .
$$

Finally, Lemma 3 will be proved with the help of the famous lemma of Boole [3] and Loomis [9], Lemma 4. Here $m$ denotes Lebesgue measure on $\mathbf{R}$.

LEMMA 4. Let $a_{1}, \ldots, a_{n}>0$ and $g(s)=\sum_{i=1}^{n} a_{i} /\left(s-t_{i}\right)$. Then

$$
m\{s: g(s)>\lambda\}=m\{s: g(s)<-\lambda\}=\frac{1}{\lambda} \sum_{i=1}^{n} a_{i} .
$$

Proof of Lemma 3. By treating the positive and negative ones separately, we may assume that all the $a_{i}$ are positive. We will count $A_{\lambda}=\left\{k: \sum_{i=-\infty}^{\infty \prime} a_{k+i} / i>\lambda\right\}$; a similar method will apply to $\left\{k: \sum_{i=-\infty}^{\infty \prime} a_{k+i} / i<-\lambda\right\}$.

Choose a finite set $A \subset A_{\lambda}$, and choose $N$ so large that $A \subset[-N, N]$ and, for each $k \in A, \Sigma_{i=-N}^{N \prime} a_{i} /(i-k)>\lambda$. Then

$$
g_{k}(s)=\sum_{i=-N}^{N}{ }^{\prime} \frac{a_{i}}{i-s}>\lambda \quad \text { for } s=k \in A,
$$

and hence $g_{k}(s)>\lambda$ for $s \in[k, k+1)$, because $g_{k}^{\prime}(s)>0$. If we let

$$
g(s)=\sum_{i=-N}^{N} \frac{a_{i}}{i-s} \text { and } h_{k}(s)=\frac{a_{k}}{k-s},
$$

then $g=g_{k}+h_{k}$, so that for each $k \in A$

$$
(k, k+1) \subset\left\{s: g_{k}(s)>\lambda\right\} \subset\left\{s: g(s)>\frac{\lambda}{2}\right\} \cup\left\{s: h_{k}(s)<-\frac{\lambda}{2}\right\} .
$$


Therefore,

$$
\begin{aligned}
\operatorname{card} A & =m\left(\bigcup_{k \in A}(k, k+1)\right) \leqslant m\left\{s: g(s)>\frac{\lambda}{2}\right\}+\sum_{k \in A} m\left\{s: h_{k}(s)<-\frac{\lambda}{2}\right\} \\
& \leqslant \frac{2 c}{\lambda} \sum_{i=-N}^{N} a_{i}+\sum_{k \in A} \frac{2 c}{\lambda} a_{k} \leqslant \frac{4 c}{\lambda}\|a\|_{1} .
\end{aligned}
$$

Proof of Lemma 2. Again we assume that all the $a_{i}$ are positive and drop the absolute value signs. Let

$$
A \subset\left\{k: \sup _{n \geqslant 1} \sum_{i=-n}^{n} \frac{a_{k+i}}{i}>\lambda\right\}
$$

be closed and bounded. For each $k \in A$ there is an interval of integers

$$
I_{k}=\left[k-n_{k}, k+n_{k}\right]
$$

such that $\sum_{i \in I_{k}}^{\prime} a_{i} /(i-k)>\lambda$. Let

$$
g_{k}(s)=\sum_{i \in I_{k}}^{\prime} \frac{a_{i}}{i-s}, \quad g(s)=\sum_{i=-\infty}^{\infty} \frac{a_{i}}{i-s}, \quad h_{k}(s)=\sum_{i \notin I_{k}} \frac{a_{i}}{i-s} .
$$

If $k \in A$, then $g_{k}(k)>\lambda$, so that either $g(k)>\lambda / 2$ or $h_{k}(k)<-\lambda / 2$. In the first case ( $k \in A_{1}$ ), by Lemma $3, k$ falls into a single (independent of $k$ ) set of measure no more than $c\|a\|_{1} / \lambda$. To deal with the left over $k$ 's $\left(k \in A_{2}\right)$, replace $\left\{I_{k}\right\}$ by a disjoint subfamily which still covers at least $\frac{1}{3}$ of $A_{2}$, by at each stage selecting an interval of maximal length disjoint from the previously chosen ones. Find $N$ such that $\cup_{k \in A_{2}} I_{k} \subset[-N, N]$ and

$$
\tilde{h}_{k}(k)<-\frac{\lambda}{2} \text { for all } k \in A_{2},
$$

where

$$
\tilde{h}_{k}(s)=\sum_{\substack{i \notin I_{k} \\ i=-N, \ldots, N}} \frac{a_{i}}{i-s} .
$$

Then also $\tilde{h}_{k}(s)<-\lambda / 2$ on $\left(k-n_{k}, k\right)$, so that we find

$$
\begin{aligned}
\operatorname{card} A & =\operatorname{card} A_{1}+\operatorname{card} A_{2} \leqslant \frac{c}{\lambda}\|a\|_{1}+6 \sum_{k \in A_{2}} n_{k} \\
& \leqslant \frac{c}{\lambda}\|a\|_{1}+6 m\left(\bigcup_{k \in A_{2}}\left\{s: \tilde{h}_{k}(s)<-\frac{\lambda}{2}\right\}\right) \\
& \leqslant \frac{c}{\lambda}\|a\|_{1}+6 m\left(\bigcup_{k \in A_{2}}\left(\left\{s: \sum_{i=-N}^{N} \frac{a_{i}}{i-s}<-\frac{\lambda}{4}\right\} \cup\left\{s: g_{k}(s)>\frac{\lambda}{4}\right\}\right)\right) \\
& \leqslant \frac{c}{\lambda}\|a\|_{1}+6 m\left\{s: \sum_{i=-N}^{N} \frac{a_{i}}{i-s}<-\frac{\lambda}{4}\right\}+6 \sum_{k \in A_{2}} m\left\{s: g_{k}(s)>\frac{\lambda}{4}\right\} \\
& \leqslant \frac{c}{\lambda}\|a\|_{1}+\frac{24 c}{\lambda}\|a\|_{1}+6 \sum_{k \in A_{2}} \frac{4 c}{\lambda} \sum_{i \in I_{k}} a_{i} \leqslant \frac{49 c}{\lambda}\|a\|_{1} .
\end{aligned}
$$


Proof of Lemma 1. By considering $f^{+}$and $f^{-}$separately, we may assume that $f \geqslant 0$. Fix $N$; we will show that

$$
\mu\left\{x: \sup _{1 \leqslant n \leqslant N}\left|\sum_{i=-n}^{n} \frac{f\left(T^{i} x\right)}{i}\right|>\lambda\right\} \leqslant \frac{c}{\lambda}\|f\|_{1},
$$

where $c$ is a constant independent of $f, \lambda$, and $N$.

For fixed $x$ and $K$, let $a_{k}=f\left(T^{k} x\right)$ and

$$
a_{k}^{K}= \begin{cases}a_{k} & \text { if }|k| \leqslant K+N \\ 0 & \text { if }|k|>K+N\end{cases}
$$

so that $\left\{a_{k}^{K}\right\} \in l^{1}$. For each $m \in \mathbf{Z}$, let

$$
G_{m}(x)=\sup _{1 \leqslant n \leqslant N}\left|\sum_{k=-n}^{n}{ }^{\prime} \frac{a_{k+m}}{k}\right|, \quad G_{m}^{K}(x)=\sup _{1 \leqslant n \leqslant N}\left|\sum_{k=-n}^{n} \frac{a_{k+m}^{K}}{k}\right| .
$$

Then

$$
\begin{aligned}
G_{m}(x) & =\sup _{1 \leqslant n \leqslant N}\left|\sum_{k=-n}^{n}{ }^{\prime} \frac{a_{k+m}^{K}}{k}+\frac{a_{k+m}-a_{k+m}^{K}}{k}\right| \\
& \leqslant G_{m}^{K}(x)+\sup _{1 \leqslant n \leqslant N}\left|\sum_{k=-n}^{n} \frac{a_{k+m}-a_{k+m}^{K}}{k}\right|,
\end{aligned}
$$

so that $G_{m}(x) \leqslant G_{m}^{K}(x)$ for $|m| \leqslant K$.

Now let $E=\left\{x: G_{0}(x)>\lambda\right\}$, so that $\left\{x: G_{m}(x)>\lambda\right\}=T^{-m} E$. Let $\bar{E}=$ $\left\{(x, m): G_{m}^{K}(x)>\lambda\right\}$. Then, if card continues to denote counting measure on $\mathbf{Z}$,

$$
\begin{aligned}
\mu \times \operatorname{card}(\bar{E}) & =\int_{X} \operatorname{card}\left\{m: G_{m}^{K}(x)>\lambda\right\} d \mu(x) \\
& \leqslant \int_{X} \frac{c}{\lambda} \sum_{m=-\infty}^{\infty}\left|a_{m}^{K}\right| d \mu \leqslant \int_{X} \frac{c}{\lambda} \sum_{-(K+N)}^{K+N}\left|a_{m}\right| d \mu \\
& \leqslant \frac{c}{\lambda}[2(K+N)+1]\|f\|_{1},
\end{aligned}
$$

and also

$$
\begin{aligned}
\mu \times \operatorname{card}(\bar{E}) & \geqslant \sum_{m=-K}^{K} \mu\left\{x: G_{m}^{K}(x)>\lambda\right\} \geqslant \sum_{m=-K}^{K} \mu\left\{x: G_{m}(x)>\lambda\right\} \\
& =\sum_{m=-K}^{K} \mu\left(T^{-m} E\right)=(2 K+1) \mu(E) .
\end{aligned}
$$

Thus,

$$
\mu(E) \leqslant \frac{c}{\lambda} \frac{2(K+N)+1}{2 K+1}\|f\|_{1},
$$

and the result follows upon letting $K \rightarrow \infty$.

A similar argument can be used to establish the continuous-parameter part of the theorem; alternatively, one may consider time $\varepsilon$ maps and approximate. 


\section{REFERENCES}

1. E. Atencia and A. de la Torre, $A$ dominated ergodic estimate for $L^{p}$ spaces with weights (preprint).

2. E. Atencia and F. J. Martin-Reyes, The maximal ergodic Hilbert transform with weights (preprint).

3. G. Boole, On the comparison of transcendents with certain applications to the theory of definite integrals, Philos. Trans. Roy. Soc. London 147 (1857), 745-803.

4. A. P. Calderón, Ergodic theory and translation-invariant operators, Proc. Nat. Acad. Sci. U. S. A. 59 (1968), 349-353.

5. M. Cotlar, A unified theory of Hilbert transforms and ergodic theorems, Rev. Mat. Cuyana 1 (1955), 105-167.

6. Alberto de la Torre, Ergodic $H^{\prime}$ spaces, Bol. Soc. Mat. Mexicana 22 (1977), 10-22.

7. R. Hunt, B. Muckenhoupt and R. Wheeden, Weighted norm inequalities for the conjugate function and Hilbert transform, Trans. Amer. Math. Soc. 176 (1973), 227-251.

8. Roger L. Jones, An analog of the Marcinkiewicz integral in ergodic theory, Studia Math. 68 (1980), 281-289.

9. Lynn H. Loomis, A note on the Hilbert transform, Bull. Amer. Math. Soc. 52 (1946), 1082-1086.

10. Norbert Wiener, The ergodic theorem, Duke Math. J. 5 (1939), 1-18.

Department of Mathematics, University of North Carolina, Chapel Hill, North Carolina 27514 